\title{
Magia e illusione nell'elaborazione di due passi centrali del Furioso
}

Come sappiamo, nell'Orlando Furioso la presenza dell'elemento magico non è sempre ovvia. $\mathrm{Ci}$ sono episodi in cui l'elemento magico è pressoché invisibile ed è generato unicamente da un peculiare trattamento inventivo-ispirativo del poeta: allora lo si deve cercare sotto la superficie. Altri episodi, invece, vanno esaminati dal punto di vista della prevalenza dell'ovviamente magico come parte di una tradizione letteraria. Le due categorie, però, spesso si sovrappongono. Anche in un episodio in cui apparentemente domina una base letteraria tradizionale, si riscontra che la propensione dell'Ariosto era di insistere non sui risultati esterni che circondano il personaggio (cioè quello che gli succede), ma sulle loro motivazioni interne.

Lo studio comparativo delle varianti delle tre successive edizioni dell'Orlando Furioso $^{1}$ può confermare e sottolineare la doppia, e opposta, tendenza ariostesca ad umanizzare, o "normalizzare," elementi magici tradizionali, e, viceversa, ad introdurre nella vicenda piuttosto ordinaria una peculiare deformazione magica. Le correzioni, quindi, non si isolano come puro, seppur interessantissimo, fatto linguistico, ma si ricollegano intimamente alla sensibilità umana, e anche poetica, dell'Ariosto. Specialmente nell'ultima redazione si percepiscono dei germi di crisi, sia dal punto di vista storico che da quello personale. La deformazione ${ }^{2}$ magica, o la soprarealtà, che opera nel poema diventa una forza coesiva fra i dati evasivi manifesti e i dati pessimistici occulti. L'elemento magico si fa allora uno strumento di discrezione ${ }^{3}$ attraverso cui il poeta può mediare tali dati pessimistici e offrire dei giudizi impliciti.

Giorgio Padoan definisce l'Orlando Furioso ". . . non tanto il poema della fantasia creatrice - come è stato detto - di uno spazio paradisiaco dove rifugiarsi dai problemi imposti dall'ingrata realtà, bensí il poema delle illusioni umane. . . ."4 Siccome lo studio della natura umana è centrale ad ogni situazione, molte 
componenti del poema suggeriscono che l'uomo preferisce l'illusione alla ragione. Per cui la magia si origina in noi ed incarna appunto la nostra preferenza per l'illusione. Gli elementi magici vanno visti, quindi, come manifestazione del bisogno dell'animo umano di procedere oltre la meschinità della realtà quotidiana ed oltre i limiti che spesso sono imposti dalla ragione. La vita, nel Furioso, si forma e si sostanzia di una ricerca continua. L'illusione e la magia diventano mezzi secondo cui l'uomo "virtuoso" allarga le sue possibilità di azione e afferma la sua vitalità in lotta contro il destino. Il trattamento dell'elemento magico è allora uno dei modi per cui l'Ariosto rivela la sua visione della vita che, spesso, appare paradossale e ambigua.

Si sa che nel Furioso viene rappresentata la vanità di gran parte dell'attività umana. Eppure il poeta, conscio dei contrasti e delle complessità inerenti alla nostra natura, non si limita meramente a condannare l'illusione che guida la ricerca vitale. Ma attraverso il lavorio del poema crea un notevole equilibrio fra l'illusione e la delusione che non distrugge, anzi genera, il dubbio che nell'uomo anche la grandezza abbia per fondamento una fiducia o speranza che inizialmente non sembra altro che irrazionalità o follia.

Un'investigazione delle varianti porta a risultati particolarmente felici proprio perché nel Furioso il poeta non rivela se stesso autore in maniera diretta: dissimula la propria persona in vari modi, come, per citare il caso piú evidente, il sotterraneo fluire dell'ironia. Qui, attraverso l'osservazione del delicato lavoro testuale, si spera di intravedere alcune istanze della "presenza" del poeta.

Uno degli episodi in cui prevale un trattamento inventivoispirativo dell'elemento magico è la fuga di Angelica. Qui la necessità di ricercare l'elemento magico sotto la superficie coincide con la scoperta che quello che veramente interessava all' Ariosto di raccontare in questi versi era "non ciò che accade ad Angelica, ma ciò che accade in Angelica." 5

Nonostante il terrore da cui si trova avviluppata, Angelica si rivela, per tutta la fuga, donna previdentissima. Dall'inizio, si affida alle proprie intuizioni ("presaga che quel giorno esser rubella / dovea Fortuna alla cristiana fede," I, 10: 5-6) e, abbandonando il campo cristiano, sa svolgere l'urgenza della situazione immediata a suo favore. E poi piú tardi, approfittandosi del duello in atto fra Rinaldo e Ferraú, Angelica volterà ancora le spalle, prendendo cosí, abilmente, l'occasione del momento: 
Or, mentre l'un con l'altro si travaglia, bisogna al palafren che 'l passo studi; che quanto può menar de la calcagna, colei lo caccia al bosco e alla campagna. (I, 17: 5-8)

Con l'ottava $33^{\mathrm{a}}$ si pone in corso il processo fortunato per cui Angelica saprà immedesimarsi con la natura che, prima, l'aveva terrorizzata. Dopo il vivace trambusto dei due cavalieri combattenti (ott.17 e seg.), ci si interna, all'ottava $33^{a}$, in una natura maestosa e oscura, fatta di misteriose sensazioni, visive, uditive e tattili. Si sente, all'ottava $33^{\mathrm{a}}$, una distinta alterazione di atmosfera:

Fugge tra selve spaventose e scure, per lochi inabitati, ermi e selvaggi. Il mover de le frondi e di verzure, che di cerri sentia, d'olmi e di faggi, fatto le avea con subite paure trovar di qua di là strani viaggi; ch'ad ogni ombra veduta $o$ in monte $o$ in valle, temea Rinaldo aver sempre alle spalle. (I, 33)

All'ottava $35^{\mathrm{a}}$ Angelica, introducendosi nel "boschetto adorno," scopre quell'insieme di qualità speciali per cui il suo terrore potrà finalmente mutarsi in esaurimento:

Quel dí e la notte e mezzo l'altro giorno s'andò aggirando, e non sapeva dove. Trovossi al fine in un boschetto adorno, che lievemente la fresca aura muove. Duo chiari rivi, mormorando intorno, sempre l'erbe vi fan tenere e nuove; e rendea ad ascoltar dolce concento, rotto tra picciol sassi, il correr lento. (I, 35)

La sua fuga conduce Angelica per caso nel "boschetto adorno," ma è lei che, guardandosi intorno, conferisce un significato speciale a quello che vede, cosicché la natura circostante "si carichi"6 dei sensi piú intimi della donna. Angelica si vede apparire davanti un mondo incantato. L'effetto di sinestesia coinvolge simultaneamente le varie modalità sensoriali della vista, del tatto e dell'udito. È stato osservato da Fredi Chiappelli che l'Ariosto concepí questa scena

in un complesso di sensazioni distinto da un aura soprannaturale. La base magica non è però manifesta: tende ad esprimersi come un carattere 
insito e onnipresente nella scena . . . il poeta suggerisce un insinuarsi repentino dell'incanto nelle fibre irrazionali dell'essere, evitando di ricorrere a meccanismi negromantici od a sortilegi dichiarati. ${ }^{7}$

È, infatti, la convinzione illusoria di Angelica di essere sicura quella che le permette di interrompere la fuga. Qui Angelica preferisce l'illusione: la convinzione di essere sicura risulta dalla propria predisposizione a crederla. L'aura soprannaturale che colorisce e informa il complesso degli effetti ottici e acustici è come un'estensione concreta dell'interno di Angelica, una scelta che viene compiuta nel subconscio quando si son fatti evidenti i limiti della ragione.

Nella $36^{a}$, e specialmente nella $37^{a}$ ottava, l'Ariosto fa evolvere ancora questo senso illusorio di un ambiente magicamente immune. Nella $36^{a}$ ottava cominciano a palesarsi le prime reazioni di Angelica di fronte al "boschetto adorno." Va attuandosi un processo di ipnosi, il cui potere suggestivo condurrà Angelica al sonno (I, 38). L'ottava $36^{\mathrm{a}}$ si presenta cosí nella redazione del 1516 , che qui e in seguito indicheremo come $A$ (le redazioni del 1521 e 1532 si indicheranno, rispettivamente, come $B$ e $C$ ):

Quivi parendo a lei d'esser sicura e lontana a Rinaldo mille miglia, da la via stanca e da la estiva arsura, di riposare alquanto si consiglia: smonta tra' fiori alla fresca verdura, et al suo palafren lieva la briglia e lo lascia nel margine de l'onde, che di fresca erba avea piene le sponde. (I, 36, A)

Nelle redazioni $B$ e $C$ invece si presenta cosí:

Quivi parendo a lei d'esser sicura e lontana a Rinaldo mille miglia, da la via stanca e da l'estiva arsura, di riposare alquanto si consiglia: tra' fiori smonta, e lascia alla pastura andare il palafren senza la briglia;

e quel va errando intorno alle chiare onde,

che di fresca erba avean piene le sponde. (I, 36, BC)

1 di $B$

3 la $B$

6 andar $B$

I primi quattro versi della $36^{\mathrm{a}}$ ottava costituiscono una parentesi in cui apparentemente si mantengono in Angelica le facoltà razio- 
nali: una breve pausa durante la quale Angelica dovrà giustificare dentro di sé il proprio impulso ad interrompere la fuga. La relazione che già si è sviluppata nell'ottava precedente fra la virtú speciale del luogo e Angelica diventa esplicita nei primi due versi della $36^{\mathrm{a}}$ ottava: "Quivi parendo a lei d'esser sicura / e lontana a Rinaldo mille miglia, /." In apparenza questi versi traducono in termini razionali i sentimenti di sollievo che hanno colpito Angelica al suo primo respiro nel "boschetto adorno." In realtà, persiste una quota sostanziale di pura emozione che si scopre nel verbo parere (v. 1) e nell'iperbolico mille miglia (v. 2). La trasparenza dell'elemento emotivo, prima, fissa in Angelica l'origine della facoltà incantatrice del luogo e, poi, allude alla conseguente illusorietà della sua convinzione di essere sicura. Primo esito di questa convinzione è il permettersi da parte di Angelica la registrazione cosciente della propria estenuazione ("da la via stanca e da l'estiva arsura, / di riposare alquanto si consiglia: /," vv. 3-4). Si è ormai concluso il legame di causa ed effetto fra il primo verso ("Quivi parendo a lei d'esser sicura") e il quarto verso ("di riposare alquanto si consiglia").

A cominciare dalla seconda metà della $36^{a}$ ottava i pensieri di Angelica non sono piú esposti in maniera esplicita. Come per sottolineare il suo stato ipnotico, l'interno di Angelica ormai si paleserà solo attraverso le sue azioni. Tale descrizione dall'esterno, però, risulterà non in una diminuzione ma in un aumento d'interiorità per la protagonista. La virtú sottile del luogo opera nel subconscio di Angelica e non si distingue piú l'intervallo fra il pensiero e la sua esecuzione: a questo punto dire quello che Angelica fa e non quello che sente diventa il modo piú autentico per rivelare il suo interno. ${ }^{8}$ Che cosa potrebbe meglio confermare l'insinuarsi dell'incanto in Angelica se non la sua irrevocabile azione di smontare dal cavallo ("tra' fiori smonta, e lascia alla pastura / andare il palafren senza le briglia; /," vv. 5-6)? Non solo, ma Angelica pure lo lascia andare in libertà, senza la briglia (v. 6). ${ }^{9}$ I cambiamenti apportati dall' Ariosto nella seconda metà della $36^{a}$ ottava rivelano la sua preoccupazione di segnalare ancora la relazione speciale che si stabilisce fra Angelica e il "boschetto adorno." Il carattere conclusivo dei due verbi smonta e lascia (v. 5) viene reso cospicuo nella terza stampa sia dalla loro posizione ora centrale nel verso che dalla loro attiguità l'uno rispetto all'altro. Come è stato osservato da Gianfranco Contini: "Mettere a fuoco l'immagine significa, per l'Ariosto, propriamente rassodare il mezzo del verso, isolarvi una nuova entità ritmica, trovare un tempo forte di piú. . . ." Esteriormente i versi 7-8 ("e quel va errando intorno 
alle chiare onde, / che di fresca erba avean piene le sponde. /" esprimono nozioni che riguardano ancora il cavallo; funzione piú vera, però, suggerita dalla loro elaborazione, sembra essere quella di ribadire certi aspetti della natura speciale del luogo introdotti nella $35^{\mathrm{a}}$ ottava. Da immagine statica nelle prime due redazioni ("E lo lascia nel margine de l'onde") il verso 7 si trasforma nell'ultima stampa in rappresentazione dinamica che si incentra sul movimento sinuoso sia del cavallo che delle "chiare onde": "e quel va errando intorno alle chiare onde." Nella $35^{a}$ ottava la coppia mormorando intorno (v. 5) si riferiva al corso dei due ruscelli; nella $36^{\mathrm{a}}$ ottava è il cavallo che "va errando intorno alle chiare onde" (v. 7): il verbo errare sembra alludere all'illusorietà del rifugio mentre intorno rammenta il serpeggiare dei ruscelli e con ciò l'idea di uno spazio "scritto" (segnato da una linea in qualche modo magicamente significativa). La sostituzione di avean ad avea (v. 8) rende conto del fatto che la linea che impronta e circoscrive lo spazio è doppia e perciò offrirebbe un campo intermedio di immunità.

L'intera $37^{a}$ ottava consiste di una descrizione del "bel cespuglio" scorto da Angelica non lontano dai due corsi d'acqua. Ma la descrizione non è obiettiva: al contrario, si esprime tutta dal punto di vista di Angelica e si ravvisano nella precisazione delle caratteristiche del cespuglio i motivi intimi che determinano le reazioni della donna. Il cespuglio diventa l'occasione per cui si conchiuderà il processo d'incantamento con l'addormentarsi di Angelica. La $37^{\mathrm{a}}$ ottava si presenta cosí nell'edizione del 1516:

Non men ch'al suo cavallo a sé provede, e mira intorno ove piú agiata pose.

Ecco non lungi un bel cespuglio vede, di spin fiorito e di vermiglie rose, ch'in modo di spelonca in sé conciede ombroso albergo ne le parti ascose: e la foglia con rami in modo è mista, ch'el sol non v'entra, non che minor vista. (I, 37, A)

Nelle ultime stampe invece si presenta cosí:

Ecco non lungi un bel cespuglio vede di prun fioriti e di vermiglie rose, che de le liquide onde al specchio siede, chiuso dal sol fra l'alte quercie ombrose; cosí voto nel mezzo, che concede fresca stanza fra l'ombre piú nascose: e la foglia coi rami in modo è mista, che 'l sol non v'entra, non che minor vista. (I, 37, BC) 


\section{2 spin $B$ \\ 5 vuoto $B$ \\ 6 capace $B$ \\ 7 con $B$}

Nelle redazioni $B$ e $C, l^{\prime}$ Ariosto inizia la $37^{a}$ ottava con il terzo verso della redazione $A$; vengono scartati i primi due versi originali che in effetti stonavano per il distacco creato dal loro carattere didascalico e troppo scopertamente razionale ("Non men ch'al suo cavallo a sé provede, / e mira intorno ove piú agiata pose. /," 37: $1-2, A)$. Con l'esclamativo "Ecco non lungi un bel cespuglio vede / di prun fioriti e di vermiglie rose /" $(37: 1-2, B C)$ si ha invece il senso di un'apparizione inattesa: il cespuglio si offre come il nuovo strumento per cui si sostiene e si compie il senso di un ambiente magicamente immune. Il fantasma inventivo è identico nelle redazioni finali dell'ottava, ma si acuisce e il rapporto fra l'invenzione e l'espressione viene perfezionato. Sono i versi 3-4 in cui si trovano le componenti nuove che l'Ariosto, avendo eliminato i primi due versi originali, aggiunse nelle ultime stampe. Nel terzo verso ("che de le liquide onde al specchio siede") l'autore precisa una nozione generica introdotta nel primo verso dell'ottava: allora fu detto semplicemente che il cespuglio non si trovava lontano dai due ruscelli. Si stabilisce ora invece una relazione diretta fra i corsi d'acqua e il cespuglio: "che de le liquide onde al specchio siede." L'immagine dello specchio (v. 3) suggerisce non solo un nesso ma una corrispondenza reciproca fra le acque e il cespuglio: va dilatandosi dunque, con la partecipazione speculare del cespuglio al prodigio delle acque, l'influsso della virtú sottile del luogo. I corsi d'acqua formano due sinusoidi orizzontali che circoscrivono lo spazio. Nel quarto verso ("chiuso dal sol fra l'alte quercie ombrose") si definisce una linea verticale, prodotta da l'alte quercie ombrose (v. 4): il cespuglio è chiuso dal sol (v. 4). L'ombra delle querce serve da oscuro velo sopra il cespuglio; poi, a sua volta, il cespuglio farà da ombrello sopra Angelica. Al primo colpo d'occhio il cespuglio attrae per i suoi prun fioriti e le sue vermiglie rose (v. 2). Vero suo pregio, però, dalla prospettiva di Angelica, è la sua capacità di nascondere colui che ci si addentra: "cosí voto nel mezzo, che concede / fresca stanza fra l'ombre piú nascose l" (vv. 5-6). Domina dal quarto verso il senso di un progressivo insinuarsi nella scena di ombre e di tenebre che finiscono per avviluppare tutto lo spazio. I raggi del sole sono ostacolati sia dalle querce (v. 4) che dal cespuglio stesso: "e la foglia coi rami in modo è mista, / che 'l sol non v'entra, non che minor vista. l" (vv. 
7-8). L'ambiente è diventato un involucro: a fasciare lo spazio sono due linee orizzontali (i due ruscelli) e due linee verticali (il cespuglio con sopra le querce). Si è tessuta una specie di crisalide, in mezzo alla quale si immergerà Angelica (I, 38):

Dentro letto vi fan tenere erbette, ch'invitano a posar chi s'appresenta. La bella donna in mezzo a quel si mette; ivi si corca, et ivi s'addormenta. (I, 38: 1-4)

Come sappiamo, però, Angelica si sveglia presto: arriva Sacripante e con lui il calpestio (I, 38: 6) per cui si dilegua l'incanto del dolce concento (I, 35: 7). Angelica riacquista subito la propria coscienza e riprende a riflettere e a calcolare come sfruttare l'occasione (I, 50: 5) offertasi con l'arrivo del cavaliere: ". . . alcuna finzione, alcuno inganno / di tenerlo in speranza ordisce e trama" (I, 51: 5-6). Agli interminabili gridi lamentosi di Sacripante contro la Fortuna crudel (I, 44: 3), Angelica rimane "dura e fredda piú d'una colonna" (I, 49: 5). Questa volta Angelica non subisce l'incanto, ma decide piuttosto, con tutta razionalità, di crearne un altro: creerà l'incanto di sé. All'improvviso esce dal cespuglio, appare come dea ad un Sacripante meravigliato:

E fuor di quel cespuglio oscuro e cieco

fa di sé bella et improvisa mostra, come di selva o fuor d'ombroso speco

Diana in scena o Citerea si mostra;

e dice all'apparir: - Pace sia teco;

teco difenda Dio la fama nostra,

e non comporti, contra ogni ragione,

ch'abbi di me sí falsa opinione. (I, 52)

Infine il rifugio si è rivelato illusorio; Angelica ne è emersa, però, rinvigorita: l'illusione le è servita come pausa ristorativa.

Nel Furioso la vita, che si ricrea sotto veste fantastica, diventa anche pura energia psichica: la ricerca, smaniosa, non si risolve mai. È questa, forse, la nozione che accorda, e in un certo senso confonde, i vari livelli di un episodio come quello di Ruggiero nell'isola di Alcina. L'episodio è infuso di elementi letterari tradizionali ${ }^{11}$ e di significati allegorici, eppure, anche qui, ci si avvede che il tutto non è che una proiezione all'esterno del fatto intimo. 
Ruggiero è il personaggio che rappresenta al massimo l'impulso umano di sprofondarsi in illusioni. ${ }^{12}$ Ed è, anche, fra tutti, il personaggio piú legato ad un destino inflessible ed inevitabile: come capostipite degli Estensi, dovrà convertirsi al cristianesimo e morire giovane per tradimento dei Maganzesi. Pensando a Ruggiero, Italo Calvino scrisse:

Duro destino è l'avere un destino. L'uomo predestinato avanza e i suoi passi non possono portarlo che là. . . . Sappiamo bene che tutti gli ostacoli saranno vani . . . ma ci resta il dubbio se ciò che veramente conta sia il lontano punto d'arrivo ... oppure siano il labirinto interminabile, gli ostacoli, gli errori, le peripezie che danno forma all'esistenza. ${ }^{13}$

Ruggiero oscilla di continuo fra due termini, uno stabilito da Bradamante e l'altro dal mago Atlante. Il cavaliere, apparentemente, vive in balia dell'urtarsi di queste due potenze contrarie. Invano, Atlante cerca di sottrarre Ruggiero al suo fiero destino (IV, 30: 5) ("et io, che l'amai sempre piú che figlio, / lo cerco trar di Francia e di periglio. I," IV, 30: 7-8); perfino l'inaccessibile castello d'acciaio che si erge sui Pirenei è frutto del superchio amore (VII, 43: 2) che il mago nutre per il suo cavallier gentil (IV, 29: 6) ("La bella ròcca solo edificai / per tenervi Ruggier sicuramente, l," IV, 31: 1-2). Si sa che alla fine il destino, e quindi Bradamante, dovrà prevalere. $\mathrm{Ma}$, come nota Calvino, Ruggiero "non sembra aver fretta" di incontrare il suo destino (". . . per ora la disciplina militare saracena e l'affetto protettivo di Atlante hanno su Ruggiero almeno altrettanto ascendente che il richiamo dell'amore per la bella nemica."). ${ }^{14}$ Il dominio di Atlante sopra Ruggiero è tale che perfino Melissa, giungendo nell'isola di Alcina, delibera di assumere le sembianze del mago ("con quello occhio pien d'ira e di minaccia, / che sí temuto già fanciullo avea; /," VII, 56: 5-6). Melissa sa che solo cosí il suo rimprovero avrà pieno effetto ("Ruggier si stava vergognoso e muto / mirando in terra, e mal sapea che dire; l," VII, 65: 1-2).

Atlante tenta con il piacere e con l'illusione. E forse Ruggiero, per il momento, si lascia ingannare proprio perché in fondo sa di non poter sfuggire al suo destino. Ruggiero, quindi, è il campione delle illusioni umane perché egli è, prima di tutto, il campione del destino ineluttabile. Viene da domandarsi se Ruggiero è attivo o passivo, se agisce sulla realtà o se subisce un mondo foggiato da altri. Ma forse le potenze rivali che si contendono Ruggiero in gran parte non sono che simboli dei drammi che tenzonano all'interno di lui. L'intero episodio dell'isola di Alcina è permeato dall'accento di una fatalità incontrastabile. E accanto all'accento di fa- 
talità, convive l'accento di una rassegnazione ironica, attraverso cui sembra venir espressa una posizione dell'Ariosto di fronte all'inevitabile. È appunto l'interazione delle due modulazioni dell'ironia e dell'inevitabilità ciò che ci permette di avvertire qui la presenza riposta dell'autore. Si deve aggiungere che qui la visione ariostesca appare particolarmente ambigua e paradossale. E cosí viene raffigurato l'uomo. Il delicato equilibrio che si crea in queste ottave fra i toni dell'ironia e dell'inevitabilità rende difficile qualsiasi discussione che vuole precisare in termini di logica la vitalità dell'episodio. L'Ariosto rappresenta degli aspetti irrazionali, e naturalissimi, della natura umana.

Nell'episodio dell'isola di Alcina, lo studio comparativo basato sulle varianti sembra acquistare un valore speciale. Nel tentativo di scoprire i vari livelli di sfumatura, si intravede la mano del poeta che compone nell'ambiguità le falde multicolori del testo. L'amore, la passione, è il grande motore dell'inevitabilità. E l'amore, attraverso l'accentuarsi di quest'inevitabilità, diventa magia. Anche nell'isola di Alcina, la magia è, appunto, fatto intimo. ${ }^{15}$ L'altra faccia della medaglia concerne il valore che assume l'ironia ariostesca in queste ottave. In parte, l'ironia sarà diretta semplicemente verso Ruggiero che incarna la propensione umana verso l'illusione. Ma l'ironia dell'Ariosto non è giudice ingenuo, bensí pone in rilievo la natura complessa della vita tutta. Sotto il velo della rassegnazione ironica il poeta sembra accennare a quel dubbio se in fondo l'essenziale non consista nel lontano punto d'arrivo ma piuttosto nelle tante peripezie che si frappongono lungo la via.

L'ottava $54^{\text {a }}$ del canto VI è uno dei culmini dell'avventura all'isola di Alcina nel senso che vi si distingue l'effettuarsi di una svolta decisiva all'interno del protagonista: siamo presaghi ormai del fatto che Ruggiero non potrà tornare indietro. Giunto sull'ippogrifo all'isola fatale (VI, 52: 2), Ruggiero comincia quasi subito a subire la potenza ammaliatrice della natura circostante. Eे sensuale e deliziosa: "culte pianure e delicati colli, / chiare acque, ombrose ripe e prati molli. I" (VI, 20: 7-8). Appena scorsa, dall'alto, l'isola, Ruggiero sente il cuore tremare piú che foglia (VI, 17: 4) per il gran spazio (VI, 19: 1), tre mila miglia (VI, 25: 8), trascorso dal grande $e$ strano augello (VI, 18: 1). Presto, perd, avvicinandosi a terra e guardandosi intorno, il cavaliere si imbeve dello stesso senso di sicurezza che hanno gli animali del luogo ("e tra quei rami con sicuri voli / cantando se ne gíano i rosignuoli. I," VI, 21: 7-8; "sicuri si vedean lepri e conigli, / e cervi con la fronte alta e superba, / senza temere ch'alcun gli uccida o pigli, l," VI, 22: 3-5): smonta in 
fretta dall'ippogrifo e si toglie le armi ("Ruggier con fretta de l'arcion si sferra," VI, 23: 3; "pose lo scudo, e l'elmo da la fronte / si trasse, e disarmossi ambe le palme; /," VI, 24: 3-4). La vulnerabilità psicologica di Ruggiero è aumentata con la distanza e la paura è servita a rendere indispensabile il sollievo che ora viene abbracciato dal cavaliere.

Ad interrompere il riposo di Ruggiero saranno i gemiti del mirto Astolfo. Il racconto di Astolfo è lungo (VI, 32-53) e, apparentemente, dovrebbe rendere Ruggiero piú forte contro le seduzioni di Alcina. Le parole del mirto, però, finiscono con una nota fatale: "Io te n'ho dato volentieri aviso; / non ch'io mi creda che debbia giovarte: I" (VI, 53: 1-2). La reazione di Ruggiero al racconto di Astolfo incomincia a esprimersi nell'ottava $54^{\mathrm{a}}$. È espressione indiretta, però, dell'autore, senza che Ruggiero apra bocca e quasi come momentaneamente assentatosi. L'ottava $54^{a}$ si presenta cosí nell'edizione del 1516 :

Ruggier, che connosciuto avea per fama

il duca Astolfo, e che sapea com'era

cugin di quella donna che tant'ama,

si dolve assai vedendo in che maniera

mutato avesse in steril pianta e grama,

per gran malía, l'umana forma vera;

e dato aiuto volentier gli arebbe,

se sapea come, tanto gli ne 'ncrebbe. (VI, 54, A)

Le edizioni del 1521 e del 1532 invece si presentano cosí:

Ruggier, che conosciuto avea per fama ch'Astolfo alla sua donna cugin era, si dolse assai che in steril pianta e grama mutato avesse la sembianza vera;

e per amor di quella che tanto ama (pur che saputo avesse in che maniera) gli avria fatto servizio: ma aiutarlo in altro non potea, ch'in confortarlo. (VI, 54, BC)

Le varianti dell'ottava $54^{\text {a }}$ mettono in evidenza la nuova vulnerabilità che si sviluppa in Ruggiero dopo che ha sentito la storia del paladino inglese. Dal punto di vista della sostanza, l'ottava $54^{\mathrm{a}}$ rimane essenzialmente uguale dalla prima all'ultima redazione: Ruggiero si duole alla vista di Astolfo cambiato in mirto, vorrebbe aiutarlo, ma non può. L'ottava viene rimaneggiata, però, di modo che si scopra in Ruggiero quella sensibilità, insieme tenue e indubbia, che infine condurrà anche lui fra le braccia della maga Al- 
cina. Ruggiero, insomma, non si ritrova piú forte dopo l'avviso di Astolfo, ma piú debole. Rispetto a Ruggiero, Attilio Momigliano ha notato che "l'esempio di Astolfo non ha operato sulla sua ra-

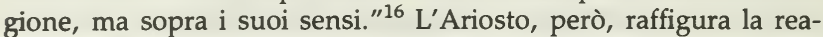
zione del cavaliere in maniera mascherata e paradossale. L'ottava $54^{\mathrm{a}}$, infatti, è problematica nel senso che i cambiamenti apportati alle ultime redazioni non risultano ciò che potrebbero apparire a prima vista. Nelle ultime stampe dell'ottava, per esempio, la presenza, e quindi l'influenza ispiratrice, di Bradamante viene apparentemente accresciuta. L'impressione originaria viene rovesciata, però, coll'evidenziarsi progressivo del principio che per Ruggiero il ricordo di Bradamante non è causa o ispirazione di forza ma piuttosto effetto, conseguenza dell'aumentata vulnerabilità del cavaliere. Ormai anche la sua donna, cioè, contribuisce alla seduzione di Ruggiero e partecipa a quella macchina fatale entro cui egli si muove. Ma insieme all'aumento di inevitabilità, si riscontra nelle ultime redazioni dell'ottava $54^{\mathrm{a}}$ un aumento dell'ironia. Nelle ultime redazioni dell'ottava $54^{a}$ il tono complessivo, invece di aggravarsi, si alleggerisce. L'elaborazione dell'ottava $54^{\mathrm{a}} \mathrm{è,} \mathrm{in-}$ fatti, esempio stupendo di come l'Ariosto, molte volte, elesse di far risplendere la sua poesia non nel dramma, né nella psicologia, ma, meravigliosamente, nell'ironia.

Nella prima redazione dell'ottava $54^{\mathrm{a}}$ il fatto che Astolfo è cugino di Bradamante viene aggiunto come corollario alle notizie strettamente personali del paladino inglese: egli è prima di tutto "il duca Astolfo," e, come tale, mantiene la propria dignità e identità individuale: "Ruggier, che connosciuto avea per fama / il duca Astolfo, e che sapea com'era / cugin di quella donna che tant'ama, /" (VI, 54: 1-3, A). Nelle ultime stampe, invece, Astolfo è diventato meramente il cugino di Bradamante: "Ruggier, che conosciuto avea per fama / ch'Astolfo alla sua donna cugin era, l' (VI, 54: 1-2, $B C)$. Scorrendo la redazione $A$ dell'ottava $54^{a}$ dal principio alla fine, il lettore avverte un impeto, o urgenza, che manca nelle redazioni che seguono. Nonostante la loro sintassi parentetica, i primi sei versi della redazione $A$ vanno letti di seguito. Sentiamo che la meraviglia e il dolore di Ruggiero sono ancorati nella realtà: egli serba ancora un senso di distacco che gli permette di farsi indietro ad osservare gli effetti della metamorfosi (vedendo in che maniera / mutato avesse. . .); Ruggiero si ferma pure a contemplare la causa (per gran malía) del misfatto, mantenendo cosí quella distanza necessaria alla propria libertà spirituale. L'insistente costruzione parentetica di questi versi ("si dolve assai vedendo in che maniera / mutato avesse in steril pianta e grama, / per gran 
malía, l'umana forma vera; l," VI, 54: 4-6 A) denota la natura ancora riflessiva delle considerazioni di Ruggiero. Nella redazione $A$ vengono precisati i particolari che indicano una comprensione razionale da parte del cavaliere del legame consecutivo fra causa ed effetto. Nelle redazioni $B$ e $C$, invece, la posizione malsicura di Ruggiero si manifesta in maniera piú netta. Al principio, data la costruzione piú schietta delle ultime redazioni dell'ottava, Ruggiero sembra perfino piú razionale di prima. L'azione ora è piú compressa, i tempi sono ristretti, e lo sgomento di Ruggiero viene espresso in maniera piú scorrevole; manca adesso l'indugio nei versi centrali dell'ottava ove Ruggiero si era fermato a considerare con meraviglia "in che maniera" Astolfo era stato trasformato in mirto. In realtà, però, lo spirito piú pratico delle redazioni finali dell'ottava $54^{a}$ sembra smentire l'apparente razionalità del protagonista alludendo ad un atteggiamento quasi troppo posato da parte di Ruggiero. Lo straordinario viene accettato quasi troppo facilmente, come se Ruggiero si fosse già fuso col mondo magico che gli è d'intorno. Ormai la trasformazione di Astolfo in mirto si riduce a semplice fatto, non si interrogano piú le sue origini, e la sostituzione di la sembianza vera a l'umana forma vera sembra adombrare una coscienza meno profonda del significato della metamorfosi: "si dolse assai che in steril pianta e grama / mutato avesse la sembianza vera; l" (vv. 3-4, BC). Già si è sbiadita nella memoria del cavaliere l'immagine naturale della "umana forma."

La prima redazione dell'ottava $54^{\text {a }}$ si chiude con una nota di vero dispiacere: " $\mathrm{e}$ dato aiuto volentier gli arebbe, / se sapea come, tanto gli ne 'ncrebbe. I" (vv. 7-8, A). E conclusione naturale che segue coerentemente i primi sei versi dell'ottava e che si impernia sul desiderio di Ruggiero di aiutare Astolfo. Con la forza dei termini volentieri e increscere si sente palpitare ancora nel dolore di Ruggiero il polso del proprio vigore. Nelle ultime redazioni non solo i due versi conclusivi ma l'intera seconda metà dell'ottava si incentra sull'aiuto che Ruggiero vorrebbe dare ad Astolfo; l'enfasi, però, è spostata:

e per amor di quella che tanto ama (pur che saputo avesse in che maniera)

gli avria fatto servizio: ma aiutarlo

in altro non potea, ch'in confortarlo. (vv. 5-8, BC)

I versi non si incentrano piú sul dispiacere da parte di Ruggiero per la metamorfosi di Astolfo. Quello che prende rilievo adesso è il vincolo di parentela fra Astolfo e Bradamante. Nelle ultime re- 
dazioni, l'ottava si divide simmetricamente in due parti, con Bradamante che domina sia la prima che la seconda. Due volte, una ai versi $1-2$, e l'altra al verso 5 , Bradamante viene rammentata esplicitamente come forza motrice: lei sola dà motivo a tutta la buona volontà di Ruggiero. Ma insieme a questa presenza piú espressa della donna, esiste nelle ultime stampe dell'ottava una nuova enfasi sull'impotenza assoluta di Ruggiero. La costruzione ipotetica di questi versi, e specialmente quella parentetica che isola il verso 6 , rende evidente il carattere vano di qualsiasi intervento da parte di Ruggiero a favore del paladino inglese. La parentesi che racchiude il verso $6 \mathrm{ci}$ fa sentire chiara la voce del poeta che osserva a distanza, lasciando il proprio suggello attraverso un'ironia vibrante. L'intera ottava, in fondo, conduce gradualmente ai due punti che si trovano in mezzo al verso 7 , e che poi servono da introduzione a quello che è il vero nucleo del discorso: "ma aiutarlo / in altro non potea che in confortarlo. /" (vv. $7-8, B C)$. Persiste il tema del "conforto," e con ciò quello dell'impotenza di Ruggiero, nel primo verso dell'ottava $55^{\mathrm{a}}$ : "Lo fe' al meglio che seppe; e domandolli / poi se via c'era. . . ." La frase Lo $f^{\prime}$ al meglio che seppe non è particolarmente promettente; ed essendo seguita da un punto e virgola, ha l'impronta della finalità: sembra accennare ad una voglia illusoria da parte di Ruggiero di poter dissolvere l'affanno di Astolfo attraverso un semplice gesto di conforto. Il primo verso della redazione $A$ dell'ottava $55^{a}$ si presenta invece cosí: "Gli rese molte grazie, e domandolli." Ruggiero dimostra qui di capire il pericolo in cui pure lui rischia di trovarsi e con i ringraziamenti viene riconosciuto anche l'obbligo che ormai sente verso il paladino inglese.

Forse l'atteggiamento che riassume meglio i cambiamenti apportati alle ultime redazioni dell'ottava $54^{\mathrm{a}}$ è la mancanza di meraviglia che avvertiamo in Ruggiero. Al di sotto del significato piú esterno dei versi, quello che il poeta sembra aver infuso nelle ultime stampe dell'ottava è la sensazione quasi di calma che, diffondendosi, progressivamente prende il posto dello stupore. Ruggiero serba ancora l'apparenza di una razionalità superiore alle circostanze, ma, in realtà, il suo cedimento è imminente. Ciò che viene perfezionato attraverso l'elaborazione dell'ottava $54^{\mathrm{a}}$ è il rapporto fra l'ironia e l'inevitabilità. Il ricordo di Bradamante allude alla nuova vulnerabilità di Ruggiero. L'ottava $54^{\mathrm{a}}$, quindi, si impernia sul paradosso. Nell'isola di Alcina l'illusione diventa destino e il destino diventa illusione.

Sia Angelica che Ruggiero hanno preferito l'illusione. Angelica ne è emersa rinvigorita e l'illusione le è servita come pausa ristora- 
tiva. Ruggiero, momentaneamente, ha trovato nell'illusione la speranza. L'illusione che spera non ammette che uno subisca con passività. Bisogna agire: le possibilità sono aperte e il destino non è ancora segnato. L'ironia, la presenza del poeta nella sua opera, fa respirare in ognuno di noi la coscienza che ebbe l'Ariosto di quella ricerca, fatta di ambiguità, che è poi la vita stessa.

\section{University of Southern California}

\section{NOTE}

1 Ludovico Ariosto, "Orlando Furioso": secondo la stampa del 1532, con le varianti delle redazioni del 1516 e del 1521, a cura di Santorre Debenedetti e Cesare Segre (Bologna: Commissione per i Testi di Lingua, 1960) (In seguito le citazioni ariostesche saranno tratte dalla suddetta edizione).

2 Cfr. Walter Binni, Metodo e Poesia di Ludovico Ariosto (Messina: G. D'Anna, 1947). Il Binni parla della ". . .'deformazione' tanto cara ai quattrocentisti per superare la bruta realtà [p. 106] . . . l'alterazione di misure comuni non per trovata intellettualistica ma in rapporto a misure piú intime che nei grandi non mancano di legare in profondo col senso piú concreto della realtà . . ." (pp. 116-17).

3 A proposito del rapporto fra le correzioni dell'Orlando Furioso e la "discrezione" ariostesca, si veda: Gianfranco Contini, "Come lavorava l'Ariosto," in Esercizi di lettura (Firenze: Le Monnier, 1947).

4 Giorgio Padoan, "L'Orlando Furioso e la crisi del Rinascimento," in Ariosto 1974 in America: Atti del Congresso Ariostesco - Dicembre 1974, Casa Italiana della Columbia University a cura di Aldo Scaglione (Ravenna: Longo, 1976), p. 9. E poi: "Gli accostamenti di osservazione realistica e racconto fantasioso, di evento storico e mondo magico, di oggettivo e fittizio, approdano alla dimostrazione che l'uomo non solo è vittima dell'illusione, ma preferisce cedere all'illusione rinunciando con troppa facilità alla Ragione..." (p. 9).

5 Fredi Chiapelli, "Sul linguaggio dell'Ariosto," in Atti dei Convegni Lincei: Convegno Internazionale Ludovico Ariosto (Roma: Accademia Nazionale dei Lincei, 1975), p. 39. L'analisi che faremo delle ottave $36^{a}$ e $37^{a}$ prende spunto dal suddetto studio dove viene esaminata l'elaborazione dell'ottava $35^{\mathrm{a}}$. Riguardo alla "presenza" nascosta del poeta nella sua opera il Chiappelli scrive: "Il principio è che l'Ariosto tende costantemente a situare la sua 'presenza' nella opera ad un livello non immediatamente apparente; l'artefice occulta la sua propria identificazione, persino nell'espediente malizioso per cui inserisce un sé stesso ironicamente discorsivo (ma inavvicinabile) nella tastiera tonale del poema" ( $p$. 33).

$6 \mathrm{Si}$ veda in proposito "Il "Carro" e il "Mare Amaro": Una Lettura de "I Malavoglia"," in Il Verga Maggiore: Sette Studi (Firenze: La Nuova Italia, 1968), p. 81 di Giovanni Cecchetti in cui mi pare che l'espressione "si carica" acquisti un significato speciale. Riferendosi agli "elementi esterni (brillare di stelle, suoni)," il Cecchetti scrive: ". . . questi hanno l'unica funzione di riflettere ed evocare fatti di vita emotiva, come avviene sempre nella psiche umana, e percio non esistono di per se stessi, ma diventano una cosa complessa, totalmente interiorizzata, parte integrante di quella vita emotiva. Il dato esterno, dunque, si carica spontaneamente delle speranze e delle apprensioni dei personaggi. . . ." Negli studi sul Verga, direi che il Cecchetti ha sviluppato delle basi teoriche di critica letteraria che possono essere riferite a diversi autori. Si fondono, nelle opere 
del Cecchetti, una rigorosa formazione classico-letteraria e una insolita sensibilita e acume per quanto riguarda la penetrazione della psiche umana.

7 Chiappelli, cit., pp. 37-38. A proposito dell'elaborazione dell'ottava $35^{\mathrm{a}}$, il Chiappelli osserva: "L'introduzione di 'sempre' $[v .6, C]$ produce un mutamento profondo nell'espressione della natura speciale soggiacente all'immagine. Il prodigio del verdeggiare perenne non era enunciato nella redazione con l'imperfetto ('facean') [v. 6, $A B]$ mentre ora si afferma esplicito. ...."

8 Esaminando la scena dell'abbandono di Olimpia, il Chiappelli distingue un ". . graduale ridursi dello sfasamento fra impulso ed azione. Portato il personaggio alla crisi, l'Ariosto ha già sapientemente rovesciato il procedimento. Il suo fantasma è ora un essere umano invaso da un terrore disperato, piú forte della ragione: le azioni non piú seguono, ma procedono la valutazione e l'operazione intellettiva" (Chiappelli, cit., p. 46).

9 Per uno studio sul significato nell'O.F. dell'immagine del cavallo con e senza il freno delle redini, si veda A. Bartlett Giamatti, "Sfrenatura: Restraint and Release in the Orlando Furioso," in Ariosto 1974 in America, cit., pp. 32-39: "The image of the rein and of the curbed or uncurbed horse occurs throughout Orlando Furioso in a variety of ways. . . . The idea of 'gathering in' the horse, finally of integrating and consolidating the self, is at the heart of his vision. He [Ariosto] is always concerned with the collected, as opposed to the dispersed or scattered, self. . . . The horse out of control is simply a symbol of human despair. When 'beside oneself,' 'di sé tolta,' as Angelica is, one is 'sfrenato' like the horse. How one rides is an index to one's spiritual state" (pp. 32-33).

10 Contini, cit., p. 236.

11 Delle fonti dell'episodio dell'isola di Alcina, e specificamente di Astolfo cambiato in mirto, P. Rajna, Le fonti dell' "Orlando Furioso" (Firenze: Sansoni, 2nd ed. 1900), p. 169-70, scrive: "Virgilio e Dante . . . hanno manifestamente contribuito all'invenzione (Aen., III, 22; Inf. XIII. 31). Ma anche due imitazioni nel Filocolo del Boccaccio, la fonte di Fileno e il pino di Idalagos, che oltre al manifestarci convenienze minute, un pochino sospette in un'opera di questo genere, hanno comune col mirto d'Astolfo il metterci innanzi delle vittime d'amore, e la seconda tra esse propriamente della mobilità femminile, parrebbero esser state presenti all'Ariosto."

12 Padoan, cit., p. 9: "Di questa disponibilità a lasciarsi ingannare - e molti, avverte l'autore, sono coloro che per ciò usano 'simulazion, menzogne e frodi' (VIII, 1: 7) - è esempio illustre, non il solo, lo stesso Ruggiero: il quale, pur messo in guardia contro le arti di Alcina preferisce credere che Astolfo gli abbia mentito (VII, 16-17)."

13 Italo Calvino, "Orlando Furioso" di Ludovico Ariosto raccontato da Italo Calvino Con una scelta del poema (Torino: Einaudi, 1970), p. 24.

14 Calvino, cit., pp. 24-25.

15 Del regno di Alcina, Attilio Momigliano, in Saggio su l' "Orlando Furioso" (Bari: Laterza, 1952), pp. 32-33, nota che ". . . manca di quell'appariscenza che è il carattere comune degli spettacoli magici: perché qui, come nell'intero episodio della fata, la magia è tutta interna. . . . Il giovane [Ruggiero] è prigioniero di una seduzione sensuale. . . . La magia è, piú che altro, in una leggiera accentuazione di quella fatalità che è sempre in fondo della sensualità amorosa. . . ."

16 Momigliano, cit., pp. 23-26: "Dopo l'avviso di Astolfo, l'alunno del mago non si ritrova piú forte contro le lusinghe di Alcina, come potremmo credere, ma piú debole: l'esempio di Astolfo non ha operato sulla sua ragione, ma sopra i suoi sensi. ... Anche nella pieta di Ruggiero sentite la carezza; e perfino nel ricordo di Bradamente . . . sentite l'armonia dell'impareggiabile tessuto di seduzione che vi si svolge dinanzi. L'aura dolce del luogo dispone il guerriero rinfrancato ai sentimenti affettuosi...." II Momigliano giustamente mette in evidenza le forze (la natura circostante, Astolfo, Bradamante) che, insieme, contribuiscono alla seduzione di Ruggiero. Quello che sembra mancare alla sua analisi, pero, è una considerazione del ruolo che gioca l'ironia nell'episodio del- 
l'isola di Alcina. Senza quella cadenza ironica, che contribuisce cosí tanto al tono generale dell'episodio e in cui avvertiamo la presenza del poeta, le "sensazioni dolci" (p. 31) e "l'impeto lirico" (p. 36) di cui parla il Momigliano, si appesantiscono troppo, per il gusto ariostesco, di pura intensità psicologica. Riguardo al problema generale dell'ironia nell'Ariosto, si veda lo studio "Folly in the Orlando Furioso: A Reading of the Gabrina Episode," in Forum Italicum, XIV, 1 (Spring 1980), 56-77. Qui l'ironia ariostesca viene intesa in maniera stimolante da Franco Masciandaro. Parlando del conflitto nell'O.F. fra le convenzioni social-cavalleresche e la realtà, il Masciandaro scrive: "What the poet rejects is not so much the substance of those ideals, but rather the categorical, axiomatic manner in which they are stated and prescribed. His irony is directed at the often obsessive inflexibility with which the man of single vision clings to his ideals. . . . Folly is thus identified with the failure to perceive reality as a fluid, constantly changing 'system of relationships,' and to measure one value against another, one possible choice against another" (p. 63). 